\title{
Development
}

\section{More than a Corepressor: The Role of CoREST Proteins in Neurodevelopment}

\author{
Simon Maksour, ${ }^{1,2}$ Lezanne Ooi, ${ }^{1,3}$ and Mirella Dottori ${ }^{1,2}$
}

https://doi.org/10.1523/ENEURO.0337-19.2020

${ }^{1}$ Illawarra Health and Medical Research Institute, Wollongong, New South Wales 2522, Australia, ${ }^{2}$ School of Medicine, University of Wollongong, Wollongong, New South Wales 2522, Australia, and ${ }^{3}$ School of Chemistry and Molecular Bioscience, University of Wollongong, Wollongong, New South Wales 2522, Australia

\begin{abstract}
The molecular mechanisms governing normal neurodevelopment are tightly regulated by the action of transcription factors. Repressor element 1 (RE1) silencing transcription factor (REST) is widely documented as a regulator of neurogenesis that acts by recruiting corepressor proteins and repressing neuronal gene expression in non-neuronal cells. The REST corepressor 1 (CoREST1), CoREST2, and CoREST3 are best described for their role as part of the REST complex. However, recent evidence has shown the proteins have the ability to repress expression of distinct target genes in a REST-independent manner. These findings indicate that each CoREST paralogue may have distinct and critical roles in regulating neurodevelopment and are more than simply "REST corepressors," whereby they act as independent repressors orchestrating biological processes during neurodevelopment.
\end{abstract}

Key words: CoREST; differentiation; gene expression; neurodevelopment; REST; transcription factor

\section{Significance Statement}

The molecular mechanisms governing normal development of the brain are yet to be fully elucidated. The regulation of gene expression by transcription factors plays a significant role in the specification and maturation of neurons and glia. Repressor element 1 (RE1) silencing transcription factor (REST) has been well characterized as a transcriptional regulator of neurogenesis through the formation of a complex with the REST corepressor (CoREST) proteins. Recently, the CoREST protein family has been shown to independently target unique genes, have distinct expression patterns, and important REST-independent functions during neurodevelopment. Understanding the molecular mechanisms governed by the CoREST family will provide insight into the regulatory networks directing normal neurodevelopment.

\section{Introduction}

Understanding the complex molecular mechanisms regulating gene expression in the brain is integral in providing insight into the processes that govern normal development, and conversely, are disrupted in neurologic diseases. The precise and tightly regulated differentiation

Received August 19, 2019; accepted February 5, 2020; First published February 19, 2020.

The authors declare no competing financial interests.

Author contributions: S.M., L.O., and M.D. wrote the paper.

S.M. is a recipient of an Australian Government Research Training Program Awards and L.O. is supported by the National Health and Medical Research Council of Australia Boosting Dementia Research Leadership Fellowship APP1135720. of stem cells during embryogenesis and neurogenesis is essential for cells, tissues and organs to form and function properly. Transcription factors play an important role in regulating both pluripotency and cell differentiation by controlling expression patterns of genes critical for development (Boyer et al., 2005). One transcription factor that

Correspondence should be addressed to Mirella Dottori at mdottori@ uow.edu.au.

https://doi.org/10.1523/ENEURO.0337-19.2020

Copyright (C) 2020 Maksour et al.

This is an open-access article distributed under the terms of the Creative Commons Attribution 4.0 International license, which permits unrestricted use, distribution and reproduction in any medium provided that the original work is properly attributed. 
governs pluripotency and cell fate is repressor element 1 (RE1) silencing transcription factor (REST; also known as neuron restrictive silencer factor, NRSF). Through repressing the expression of target genes, REST regulates neurogenesis, neuronal differentiation and maturation (Paquette et al., 2000; Ballas et al., 2005; Gupta et al., 2009; Gao et al., 2011; Mandel et al., 2011), in addition to a playing role in neuroprotection (Lu et al., 2014; Song et al., 2016, 2017a). Dysfunction of REST and its corepressor proteins are hypothesized to cause disruption in gene regulatory networks, contributing to the pathophysiology of neurodegenerative conditions, including Alzheimer's disease (AD; Lu et al., 2014; Ashton et al., 2017; Meyer et al., 2019), Huntington's disease (HD; Zuccato et al., 2003, 2007; Conforti et al., 2013), Parkinson's (Suo et al., 2015; Huang et al., 2019; Kawamura et al., 2019), and Prion disease (Song et al., 2016, 2017a,b).

Genome wide analysis revealed $\sim 2000$ potential REST targets genes in the human genome (Bruce et al., 2004; Johnson et al., 2006). REST represses transcription by forming a complex with the REST corepressor 1 (CoREST1) and recruiting chromatin modifying enzymes to induce a condensed chromatin state. Two paralogues, CoREST2 or CoREST3, have also been shown to form a complex with REST (McGann et al., 2014; Jung et al., 2018). However, the importance of the CoREST proteins is only just emerging, as evidence suggests they have the ability to target unique genes, in a REST-independent manner, in various neural and glial cell types at different stages of development (Abrajano et al., 2009a, b; Wu et al., 2018). The expression profile, regulatory networks and function of the CoREST family in neurodevelopment is only partially defined. In this review we discuss what is currently understood about the role of the CoREST family in neurodevelopment and how these proteins have a broader spectrum than acting solely as "REST corepressors."

\section{REST}

REST was initially discovered in 1995 as an integral component of the central nervous system through its role as a master negative regulator of neuronal gene expression (Chong et al., 1995; Schoenherr and Anderson, 1995). REST is a member of the Kruppel-type zinc finger transcription factor family, containing eight GL1 Kruppel zinc fingers in the DNA binding domain (Chong et al., 1995; Palm et al., 1999). The binding domain allows REST to bind to its target genes through the highly conserved 21 base pair DNA sequence motif, known as the RE1 site (Chong et al., 1995; Schoenherr and Anderson, 1995). Chromatin immunoprecipitation-coupled with deep sequencing (ChIP-seq) experiments have identified REST to bind with $\sim 2000$ genes within the human genome (Satoh et al., 2013; Rockowitz and Zheng, 2015) and 308 genes in neurons derived from human embryonic stem cells (ESCs) unique to the targets observed in ESCs (Satoh et al., 2013). Although the RE1 site is observed within a wide range of genes, it remains unclear whether REST interacts and represses expression at these sites in vivo.

\section{Role of REST in neurogenesis, neuroprotection, and neurodegeneration}

REST is an important regulatory factor within the developing nervous system through repressing transcription of genes associated with neuronal differentiation and maturation (Tabuchi et al., 2002; Bruce et al., 2004; Ballas et al., 2005). Through the repression of neuronal genes REST regulates the switch between precursor cell specification and differentiation, with REST expression decreasing with development allowing for neuronal maturation (Su et al., 2004; Gao et al., 2011; Kim et al., 2015; Nechiporuk et al., 2016). REST mediated gene suppression is facilitated by the recruitment of two corepressor complexes, mammalian Swi-independent 3 (mSin3) and CoREST, that allow for the binding of chromatin-modifying enzymes (Ballas et al., 2005; Inui et al., 2017). In addition to its initial role in repressing neuronal genes in nonneuronal cells such as Scn2a2 (encodes for $\mathrm{Na}_{v} 1.2$ ), Stmn2, Tubb3 (N-tubulin), Grm2 (also known as GluR2), Bdnf, and Calb1 (calbindin; Armisén et al., 2002; Kuwabara et al., 2004; Ballas et al., 2005), REST and its corepressor proteins have also been implicated in the regulation of other aspects of neurogenesis. For example, REST has been described to regulate phenotypic switches between neuronal subtypes, whereby increased levels of REST downregulate Gad1 (encoding for GAD67) and reduce PV-positive GABAergic interneurons in mice (Singh et al., 2019). REST is also responsible for somatosensory neuronal remodeling in pain states, with genetic deletion of Rest in mice effectively preventing hyperalgesia (Zhang et al., 2019). REST regulates synaptic plasticity in the rat hippocampus through the timely developmental switch in synaptic NMDA receptors (NMDARs) through the repression of Grin2 $b$, thus promoting NMDARs primarily composed of GluN2A subunits (Rodenas-Ruano et al., 2012). Other studies also provide evidence that REST plays a role in regulating the signaling cascades from neuronal insult to cell death. Under ischemic conditions REST levels are upregulated resulting in the suppression of GluR2 expression altering calcium permeability of CA1 neurons in the hippocampus thus hypothesized to affect influence neuronal survival (Calderone et al., 2003). Collectively, these studies have shown that the physiological role of REST is not only the repression of neuronal genes in non-neuronal cells but also governs broader aspects of neurogenesis and maintenance of mature neurons including regulating synaptic plasticity, neuronal remodeling and cell death.

REST also plays a role in neuroprotection, with aberrant expression or altered subcellular localization associated with a range of neurodegenerative diseases. In $A D$, there has been shown to be a decrease in REST expression in human cortical and hippocampal postmortem tissue. This finding was accompanied by a loss of nuclear REST and an upregulation of genes involved in cell death, Alzheimer's pathology and an accelerated differentiation of neural progenitors (Lu et al., 2014; Meyer et al., 2019). In addition, a decline in REST plasma levels was associated with increasing severity of risk and impairment in patients with mild cognitive impairment and AD (Ashton et al., 2017). REST has been implicated in HD as mutant Huntingtin 
protein cannot sequester REST in the cytoplasm, leading to an increase in nuclear REST in striatal neurons and the repression of the REST target gene BDNF, contributing to an increased susceptibility to neuronal cell death (Zuccato et al., 2003, 2007; Conforti et al., 2013). REST was also shown to be an essential mediator of the neuroprotective function of the histone deacetylase (HDAC) inhibitor trichostatin A (TSA) Parkinson's disease mouse model, as REST-deficient mice treated with TSA showed no improvement in dopaminergic neurotoxicity, TH and striatal BDNF levels and motor ability (Suo et al., 2015; Huang et al., 2019). The authors suggest that this effect is due to REST knock-out reducing adult neurogenesis and neural stem cell (NSC) survival (Huang et al., 2019). In human postmortem tissue, there is a loss of nuclear REST in aged dopaminergic neurons in Parkinson's disease patients and an increased accumulation of REST in Lewy bodies and pale bodies, suggesting its sequestration in aggregates may diminish neuroprotective signaling (Kawamura et al., 2019). In an infectious model of Prion disease in hamsters and in vitro cell models, REST expression decreased and there was loss of nuclear REST. Overexpression of REST protected against the neurotoxic peptide PrP106-126, induced neuronal oxidative stress, mitochondrial damage, synaptic dysfunction, and neurofibrillary degeneration, potentially through the action of the Akt-mTOR and Wnt$\beta$-catenin signaling pathways (Song et al., 2016, 2017a,b). Taken together, it is evident that REST plays a critical role in neurodevelopment, is required for normal aging and neuroprotection of the brain and exhibits region-specific and cell type-dependent effects in neurodegenerative diseases.

\section{REST-mediated gene repression}

Chromatin is a complex critical for packaging DNA within the nucleus of a cell. The base unit of chromatin is a nucleosome which is composed of eight histones that are encircled by 147 base pairs of DNA. Histones have an unstructured $\mathrm{N}$-terminal tail that allows for the regulation of transcription through changes in nucleosome-DNA interactions. Gene expression is regulated by transcription factors that activate or repress transcription through the stepwise recruitment of chromatin-modifying enzymes. Modifications of chromatin include acetylation (Allfrey et al., 1964), methylation (Allfrey et al., 1964), phosphorylation (Wei et al., 1999), sumoylation (Shiio and Eisenman, 2003), and ubiquitination (Sun and Allis, 2002).

REST exerts its repressive effects on target gene expression through recruiting two separate corepressor complexes, mSin 3 and CoREST1 (formerly known as CoREST), which in turn facilitate the binding of chromatin-modifying enzymes (Ballas et al., 2005; Yu et al., 2011; Inui et al., 2017). $m \operatorname{Sin} 3 A$ or $m S i n 3 B$ bind to the $N$ terminus of REST and recruit HDAC1 and HDAC2 (Huang et al., 1999; Naruse et al., 1999; Grimes et al., 2000). The $C$ terminus of REST binds the corepressor protein, CoREST1 (Barrios et al., 2014). Two paralogues, CoREST2 and CoREST3, have been found in humans and also form a complex with REST (McGann et al., 2014; Jung et al., 2018). However, their transcriptional activity and expression profile in the human brain remains largely unknown (Barrios et al., 2014; Sáez et al., 2015). Research suggests that each CoREST protein may play a different role in neurodevelopment via targeting unique genes in neural and glial cell types during development (Abrajano et al., 2009a,b, 2010).

In the complex with REST, CoREST proteins recruit complementary chromatin-modifying enzymes, including lysine-specific histone demethylase 1A (LSD1; also known as KDM1A), HDAC1/2, the H3K9 methyltransferase G9a, and the chromatin remodeling enzyme brahmarelated gene-1 (BRG1) to target genes in order to regulate transcription (Battaglioli et al., 2002; Roopra et al., 2004; Lee et al., 2005; Ooi et al., 2006). To induce a repressive chromatin state, first BRG1 recognizes acetylated histone 4 lysine 8 (H4K8) and stabilizes REST binding to the RE1 site within target genes (Fig. 1A; Battaglioli et al., 2002). HDAC1/2 then deacetylates H3K9 (Fig. 1B), allowing for G9a to methylate H3K9 and LSD1 to demethylate monomethylated or dimethylated H3K4 (Fig. 1C; Tachibana et al., 2001; Roopra et al., 2004; Shi et al., 2005). The recruitment of heterochromatin protein 1 (HP1) and methyl CpG-binding protein 2 (MeCP2) to the high-affinity site of methylated H3K9 causes chromatin condensation and thus represses gene expression (Fig. 1D; Lunyak et al., 2002; Fuks et al., 2003). The stepwise activity of the REST complex is integral in the regulation of neurodevelopmental processes including neurogenesis (Gao et al., 2011), neuronal differentiation and maturation (Kim et al., 2015), synaptic plasticity (Rodenas-Ruano et al., 2012), and neuroprotection (Lu et al., 2014; Song et al., 2017b). Disruptions to REST-mediated gene repression are hypothesized to result in the breakdown of these key neuronal processes and contribute to the pathophysiology of neurodegenerative conditions.

\section{The CoREST Protein Family}

The role of the CoREST family in neurodevelopment is less understood than those of REST. However, studies have indicated that CoREST proteins have distinct roles in neurogenesis, neuronal differentiation and maturation that are independent of REST. Despite their high sequence similarity in humans, evidence suggests each of the CoREST family members elicits unique functions at different stages of development (Yang et al., 2011; Wang et al., 2016; Jung et al., 2018). While the CoREST proteins appear to have independent roles, the exact function, target genes and expression pattern of each paralogue in neural and glial cells remains to be precisely defined. Biologically-relevant animal and cell-based models are essential for defining the molecular function of CoREST paralogues and providing insight into the mechanisms of neurodevelopment. To date, several different models, including rodent (Wang et al., 2016; Monaghan et al., 2017), stem cell (Yang et al., 2011), and established cell lines (Gómez et al., 2008), have been utilized to study the role of CoREST proteins in development. The current understanding of the CoREST family expression profile, target genes and functional roles in neurodevelopment are discussed in detail below. 


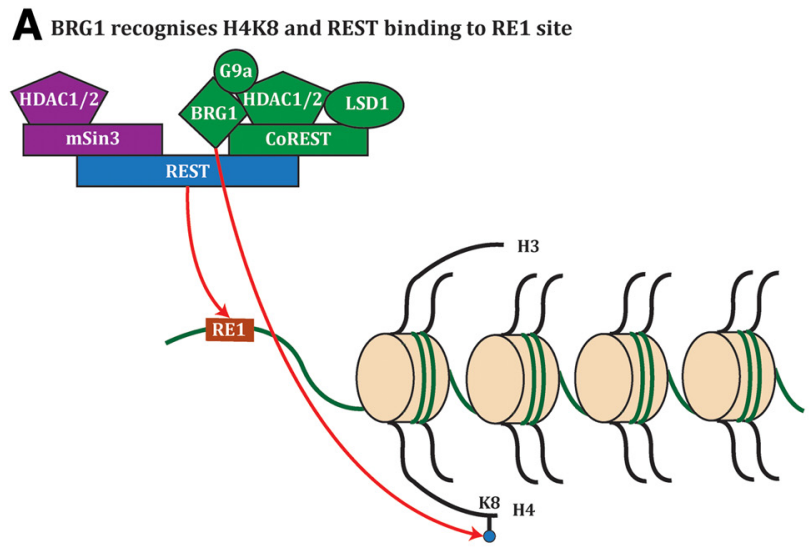

C G9a methylates H3K9 and LSD1 demethylates H3K4

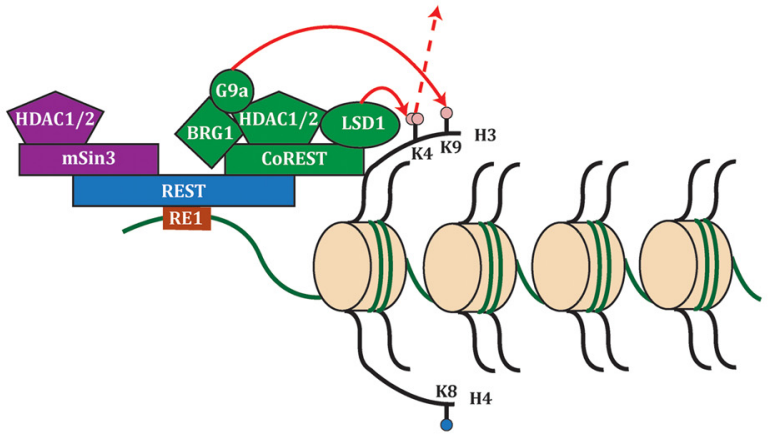

B HDAC1/2 deacetylates H3K9

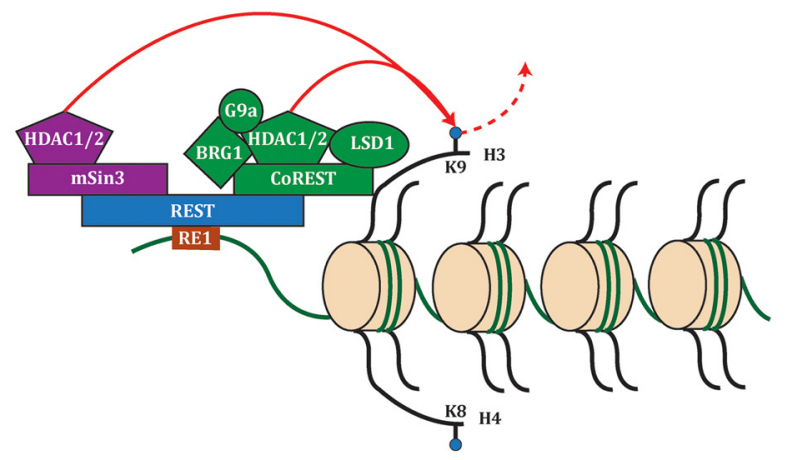

D Chromatin condensation

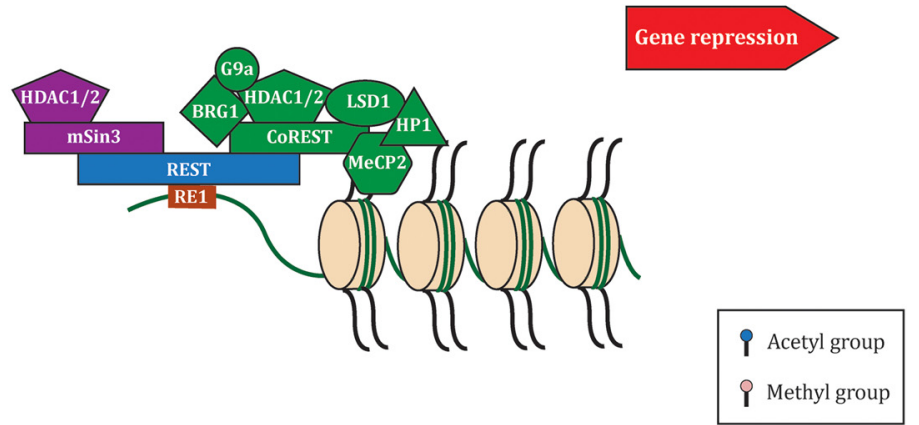

Figure 1. Schematic of REST-mediated gene repression. REST forms a complex with mSin3 (N-terminal) and CoREST (C-terminal) that in turn recruit an array of chromatin modifying enzymes. $\boldsymbol{A}$, Initially, REST binds to the RE1 site and is stabilized by the interaction between BRG1 and acetylated H4K8. B, Following on, HDAC1/2 deacetylate H3K9. C, G9a methylates H3K9 and LSD1 demethylates monomethylated or dimethylated H3K4. $\boldsymbol{D}$, Finally, chromatin is condensed via the recruitment of HP1 and MeCP2 to the high-affinity methylated $\mathrm{H} 3 \mathrm{~K} 9$, thus repressing gene expression.

\section{CoREST genes, transcripts, and protein structure}

REST corepressor 1 (RCOR1) is a 12-exon gene located on chromosome 14 that encodes CoREST1, a 53-kDa protein composed of 485 amino acids (Andrés et al., 1999). CoREST2 is a $58-\mathrm{kDa}$ protein composed of 523 amino acids expressed by REST corepressor 2 (RCOR2) which is a 13-exon gene located on chromosome 19 (Barrios et al., 2014). The final paralogue, CoREST3, is encoded by the 19-exon gene REST corepressor 3 (RCOR3) located on chromosome 1 , and is predicted to exist as four different splice variants. The variants are 48-, 50-, 56, and $61-\mathrm{kDa}$ proteins consisting of $436,449,495$, and 553 amino acids, respectively (Barrios et al., 2014).

CoREST proteins interact with the REST complex via a single zinc finger domain in the C-terminal half of REST (Andrés et al., 1999). A single point mutation resulted in abolished CoREST binding and transcriptional repression by the complex (Andrés et al., 1999). The function of the REST complex has been widely studied since discovery in 1995, with less known about the CoREST complex. Bioinformatics, structural analysis and immunoprecipitation assays of the CoREST family has provided insight into the components of the complex, how it interacts with DNA and potential mechanisms of epigenetic modifications to regulate gene expression. Each CoREST protein contains two Swi3, Ada2, N-CoR, TFIIIB (SANT) domains hypothesized to have a role in histone tail recognition and remodeling (Boyer et al., 2002, 2004; Lee et al., 2005; Shi et al., 2005) and a single Egl-27 and MTA homology 2 (ELM2) domain that acts as a protein-binding and potentially a DNA-binding site (Fig. 2; Solari et al., 1999; Lee et al., 2005; Barrios et al., 2014). The ELM2 and SANT1 domains are essential in recruiting HDAC1/2 (You et al., 2001; Ding et al., 2003; Lee et al., 2005). A nonconserved leucine at residue 165 in the SANT1 domain of CoREST2 results in impaired association with HDAC1/2 when compared with the other paralogues (Fig. 2, dotted red line; Barrios et al., 2014). The conserved linker domain between both SANT domains (Lee et al., 2005) or the SANT2 domain alone (Shi et al., 2005; Yang et al., 2006; Forneris et al., 2007) is responsible for interacting with LSD1. The SANT2 domain has been shown to mediate DNA binding (Yang et al., 2006; Pilotto et al., 2015). The shortest CoREST3 splice variant (isoform b; Fig. 2) only contains the SANT1 and ELM2 domain, limiting its ability to form a complex with LSD1, therefore reducing its transcriptional repressive capacity, and in some instances, resulting in the antagonism of CoREST1 (Barrios et al., 2014; Upadhyay et al., 2014). CoREST1 and CoREST3 isoform $\mathrm{d}$ are the only variants identified to have conserved 

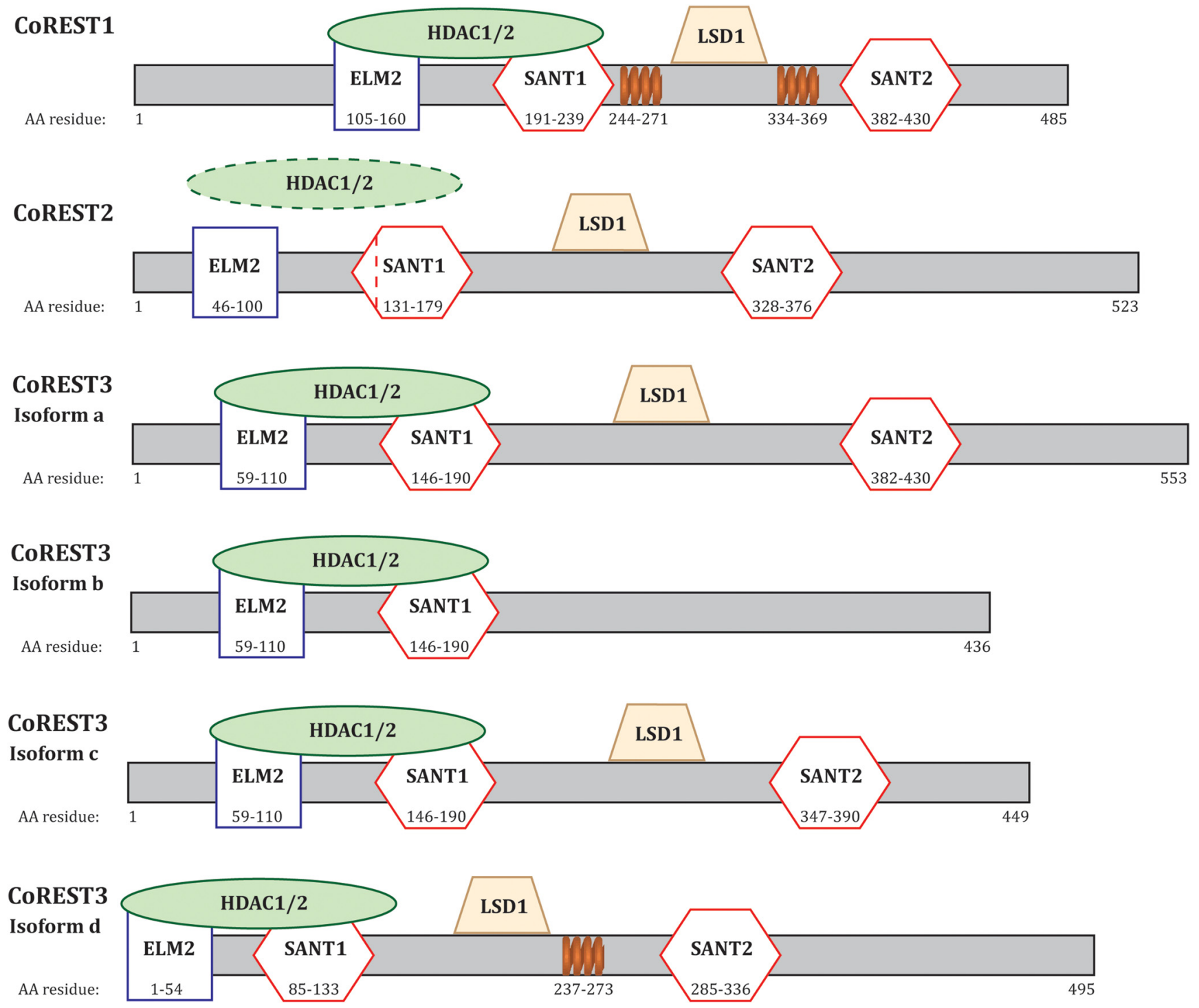

Figure 2. Structure of the CoREST proteins. Each CoREST paralogue contains an ELM2 domain and two SANT domains. The ELM2 and SANT1 domains are responsible for recruiting HDAC1/2. CoREST2 has a non-conserved leucine residue at 165 in the SANT1 domain resulting in impaired association with HDAC1/2. The linker domain between the SANT domains is responsible for binding with LSD1. CoREST3 isoform b lacks a SANT2 domain, resulting in impaired LSD1 recruitment and is responsible for the antagonistic action of the isoform. CoREST1 and CoREST3 isoform d both contain coiled-coil domains, represented by the orange coils. Information collated via UniProt Consortium (2018) and Marchler-Bauer et al. (2017).

coiled-coil domains (Fig. 2, orange coil; Marchler-Bauer et al., 2017; UniProt Consortium, 2018). Although the CoREST proteins share high sequence similarity, it may be their structural differences that result in a unique set of target genes and distinct functions in various cell types during neurodevelopment.

\section{CoREST-mediated gene repression}

CoREST proteins are best documented for their transcriptional repression ability through their interaction with REST, however, new evidence demonstrates that they have the ability to repress unique target gene expression in a REST-independent manner. The CoREST proteins elicit their transcriptional repressive ability through the formation of a complex constituted of LSD1 and the HDACs HDAC1/2 in a 1:1:1 stoichiometry, known as the LSD1-CoREST-HDAC (LCH) complex (Barrios et al., 2014; Kalin et al., 2018). The significance of each CoREST paralogue during normal development remains a novel field, with continued research certain to provide insight into the regulatory mechanisms governing neurodevelopment.

The transcriptional activity of the LCH complex is mediated by the synergistic effects of the HDAC and LSD1 enzymes (Kalin et al., 2018). The LCH complex binds to DNA through the SANT2 domain of CoREST which displaces the H3 tail (Yang et al., 2006; Pilotto et al., 2015). The DNA binding of the complex allows the histone tail to be available to the active sites of the chromatin modifying enzymes. HDAC1/2 deacetylates multiple lysine residues 

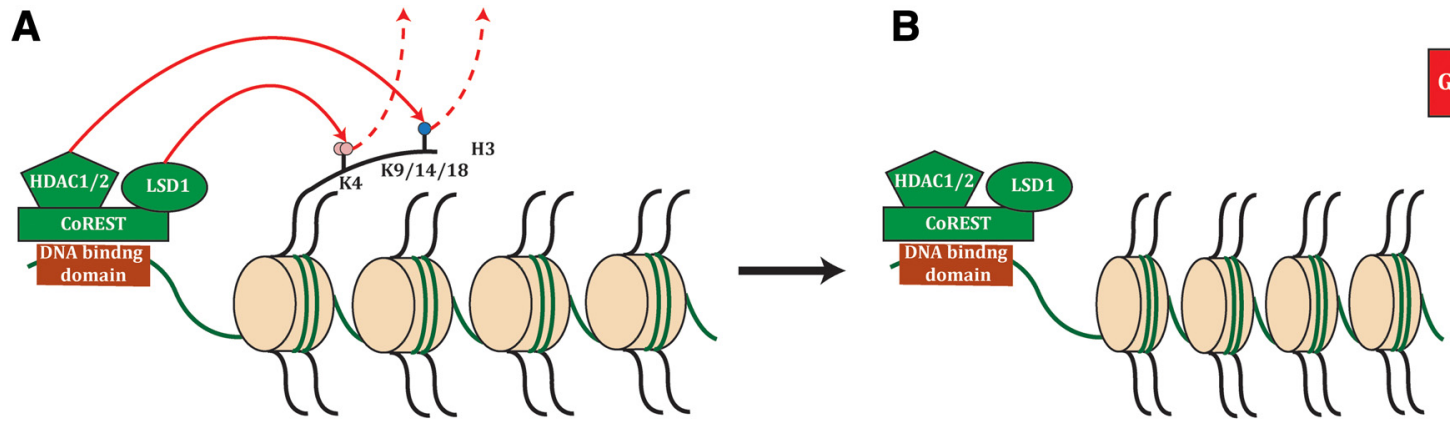

Figure 3. CoREST-mediated gene repression. CoREST forms a complex with HDAC1/2 and LSD1 to elicit transcriptional repression. $\boldsymbol{A}$, CoREST binds to DNA sites through the SANT2 domain. HDAC1/2 deacetylates multiple acetylated lysine marks on the H3 tail. LSD1 demethylates monomethylated or dimethylated H3K4. B, The synergistic function of both chromatin modifying enzymes results in chromatin condensation thus repression of gene expression.

(K9, K14, and K18) on H3 tail, while LSD1 demethylates monomethylated or dimethylated $\mathrm{H} 3 \mathrm{~K} 4$ resulting in chromatin compaction and gene repression (Fig. 3; Pilotto et al., 2015; Wu et al., 2018). The LCH demethylase activity to $\mathrm{H} 3 \mathrm{~K} 4$ is significantly inhibited by $\mathrm{H} 3 \mathrm{~K} 14$ acetylation (Wu et al., 2018). Therefore, epigenetic regulation mediated by the LCH complex will be reduced when chromatin is marked by acetylation at Lys14, leading to a diminished repressive capacity towards genes that have an abundance of acetylated H3K14 in their promoter or enhancer region (Wu et al., 2018).

The variations in the CoREST protein structures are responsible for altered protein-protein interactions and thus differences in transcriptional repressive capacity (Barrios et al., 2014). Barrios and authors provide evidence that all three paralogues behave as transcriptional repressors through luciferase reporter assays. CoREST1 exhibited the highest transcriptional repressive capacity of the three paralogues. Nucleosomal demethylation assays demonstrated LSD1 could demethylate dimethylated H3K4 in free histones, but required CoREST1 for the demethylation of nucleosomes (Upadhyay et al., 2014). CoREST2 showed similar activity to CoREST1, however with a reduced efficiency. The reduced repressive activity of CoREST3 compared with CoREST1 was not a result of diminished interaction with LSD1 but potentially from a lower catalytic efficiency (Barrios et al., 2014). In erythroid cells, the shortest isoform of CoREST3 did not facilitate nucleosomal demethylation, instead acted as an antagonist competitively inhibiting CoREST1 activity (Upadhyay et al., 2014). CoREST-mediated nucleosomal demethylation was restored by appending the SANT2 domain from CoREST1 into CoREST3. The data suggests the antagonistic and inhibitory function of CoREST3 stems from the absence of the SANT2 domain observed in the short isoform (Upadhyay et al., 2014). It also indicates that the SANT2 domain is not only required for LSD1 recruitment but additionally is crucial in mediating LCH complex nucleosomal demethylation and thus is essential in CoREST-mediated gene repression activity independent to REST. HDAC activity and coimmunoprecipitation assays in vitro revealed CoREST2 to have reduced association with HDAC1/2 when compared with its paralogues due to a non-conserved leucine residue at 165 in the SANT1 domain (Barrios et al., 2014). CoREST2 mutants that had leucine 165 modified to a serine had similar repression activity as CoREST1 and CoREST3, indicating that CoREST2 mediates transcriptional repression in a HDAC-independent manner (Barrios et al., 2014). All CoREST proteins were confirmed to interact with all splice variants of LSD1 through coimmunoprecipitation assays, suggestive of a highly adaptable LCH complex (Sáez et al., 2015). Taken together, the versatility of the $\mathrm{LCH}$ complex is indicative of a wide range of novel gene targets that may be crucial in regulating neurodevelopment. There is a prominent void in the literature regarding the differences in transcriptional repression potency and activity between the REST-CoREST and the LCH complex. Further research is required to confirm the formation of the LCH complex in vivo, characterize the DNA sequence at the binding site of the complex and thereby identify the gene targets for each CoREST protein.

\section{Expression and subcellular distribution of the CoREST proteins during neurodevelopment}

Current literature is suggestive of distinct expression profiles for each CoREST protein during neurodevelopment and in the mature brain depending on the cell type and developmental stage. The unique expression profile of each paralogue is suggestive of the formation of multiple LCH complexes, composed of a different CoREST protein core, with the potential to target a broad spectrum of target genes implicated in neurogenesis and neuronal maturation. Research has largely been based on animal studies, but provides valuable insight into the potential regulatory roles and functions the CoREST family may be involved in during neurodevelopment.

\section{CoREST paralogues exhibit an age-dependent and re- gion-specific expression pattern in the brain}

Analysis of RNA-seq databases identified widespread expression of all CoREST paralogues, including the four splice variants of CoREST3 throughout rat adult brain tissue (Sáez et al., 2015). Sáez et al. (2015) used two models 
of differentiation, nerve-growth factor (NGF)-induced neuronal differentiation of $\mathrm{PC} 12$ cells and in vitro maturation of embryonic rat cortical neurons to document changes in mRNA and protein expression of the CoREST family in neuronal maturation. CoREST1 protein levels were reduced, but RNA levels for Rcor1 remained similar throughout differentiation (Sáez et al., 2015). In addition, CoREST1 protein levels were shown to increase during embryonic development of the embryonic mouse reaching the highest levels at postnatal days 0 and 15 , followed by a reduction in the aged mouse cortex (Fuentes et al., 2012). CoREST2 mRNA levels decreased during differentiation of both PC12 cells and rat cortical neurons (Sáez et al., 2015). Additionally, CoREST2 has been shown to be highly expressed in human and mouse ESCs (Yang et al., 2011), and mRNA and protein widely expressed across most cell types of the wild-type mouse cortex (Wang et al., 2016). CoREST2 expression assessed by Western blot analysis was shown to decrease in embryonic mice brains, indicating CoREST2 may function primarily during embryonic development (Wang et al., 2016). Relative to CoREST1 and CoREST2 significantly less is currently known about the expression profile of CoREST3 during neurodevelopment. Sáez et al. (2015) concluded that CoREST3 levels remain unaltered during neuronal differentiation of both PC12 cells and rat cortical neurons. CoREST3 was also shown to be expressed in rat hippocampal, cortical and whole brain extracts via Western blot analysis (Sáez et al., 2015). As the CoREST3 expression pattern remains to be defined, knock-down and overexpression studies targeting RCOR3 will aid in identifying whether CoREST3 plays a role in regulating neuronal differentiation. Collectively, this data indicates a preliminary expression profile for the CoREST family, suggesting CoREST1 and CoREST2 levels decrease with maturation in certain brain regions, while CoREST3 expression remains unaltered in rat cortical neurogenesis. The cell types used in each model may be responsible for the variances observed in expression patterns. Further studies focusing on the expression of each CoREST paralogue during human neurodevelopment in different regions of the brain will provide insight into the functions of the CoREST family.

\section{Differential subcellular localization of the CoREST family in different cell types}

CoREST1 and REST protein expression and localization in different neuronal and glial subtypes were analyzed by immunocytochemistry and Western blotting in primary mouse neural cells (Abrajano et al., 2009a,b, 2010). Both exhibited nuclear expression in NSCs and intermediate progenitors, with expression in both the nucleus and cytoplasm of cholinergic, GABAergic, glutamatergic, and medium spiny neuron subtypes (Abrajano et al., 2009a, 2010). In glial cells, both REST and CoREST1 were expressed ubiquitously in the nucleus or cytoplasm of astrocytes and oligodendrocytes (Abrajano et al., 2009b). Immunohistochemistry of adult rat brain tissue revealed CoREST1 and CoREST2 to be expressed in the nucleus of both neurons and glial cells identified by colocalization with $\beta$-III tubulin and GFAP, respectively (Sáez et al.,
2015). CoREST3 was identified to be expressed in hippocampal, cortical tissue and mouse glial culture, suggestive of expression in both neurons and glia cell types (Sáez et al., 2015). As CoREST3 expression was described to remain unchanged during differentiation it is unclear whether the protein elicits a function. Identification of the subcellular localization of the transcription factor will provide insight into whether it is expressed in nucleus and may be potentially regulating gene expression. Collectively, the expression of CoREST1 and CoREST2 predominantly in the nucleus of both neural and glia cells is suggestive that they may be repressing gene expression by binding to chromatin in these cell types.

\section{CoREST2 expression changes throughout cell division}

CoREST2 was predominantly expressed in the nucleus in cell types of the embryonic mouse brain, however, exhibited diverse subcellular localization at different stages of the cell cycle (Wang et al., 2016). Immunohistochemical analysis showed CoREST2 in the nucleus of radial glia cells during interphase and mainly localized in chromosomes during metaphase in the ventricular zone. During anaphase, CoREST2 was partially translocated in the space between two sets of separated chromosomes (Wang et al., 2016). These findings reflect a similar pattern to LSD1 during cell cycle progression in ESCs (Nair et al., 2012). Taken together, these findings are indicative that CoREST2 may be forming a transcriptional repressive complex with LSD1 during interphase and repressing genes required for cell division and maturation.

Collectively, the expression profile and subcellular localization of CoREST proteins suggest they have the potential to regulate gene expression in both neuronal and glial cell subtypes, however, further research is required to confirm the same subcellular localization in human cells and to identify the specific target genes being repressed, further elucidating the functional roles of the CoREST family. Taken together, these findings indicate that the formation of multiple LCH complexes composed of a different CoREST protein core would broaden the genes targeted during neurodevelopment and may elicit various functions across neural and glial cell populations.

\section{CoREST proteins target unique genes compared with REST}

REST-mediated gene repression through the formation of a complex with one of the CoREST proteins is expected to target $\sim 2000$ RE1-site containing genes throughout the human genome, many essential for neuronal development (Bruce et al., 2004; Satoh et al., 2013). Research has defined a mechanism for CoREST proteins to act as transcriptional repressors independent to REST, targeting many distinct genes responsible for the modulation of neuronal and glial cell specification, maintenance, and maturation (Abrajano et al., 2009a, b, 2010; Yu et al., 2011). Genome-wide ChIP-seq in mouse ESCs investigating the binding of REST and its cofactors to sites on the genome identified CoREST1 to have 84 peaks with 61 overlapping with REST, CoREST2 to have 459 genomic binding sites and only 43 overlap with REST and 
Table 1: Summary of characterized functional roles of the CoREST family in neurodevelopment

\begin{tabular}{cc}
\hline & $\begin{array}{c}\text { CoREST pro- } \\
\text { tein involved } \\
\text { Regulation of } \\
\text { pluripotency }\end{array}$ \\
\end{tabular}

\section{CoREST3}

Regulation of neuronal differentiation and maturation

Regulation of neuroinflammation
CoREST2

CoREST1 and CoREST2

CoREST1

CoREST1 and
CoREST2

CoREST1

CoREST2

\section{Functional role}

Rcor2 knock-down resulted in reduced proliferation and impaired pluripotency; the overexpression of CoREST2, together with Oct3/4, KIf4, c-Myc, was successfully used to replace Sox2 in the generation of mouse and human induced pluripotent stem cells

$R C O R 3$ knock-down resulted in significant upregulation of NANOG and enriched acetylated $\mathrm{H} 3 \mathrm{~K} 9$ residue on the REST binding site in the NANOG promoter region; indicating CoREST3 regulates NANOG expression through the formation of a complex with REST and the deacetylation of the NANOG promoter region

Rcor1 knock-down resulted in impaired radial migration of cortical pyramidal neurons in the developing cerebral cortex; Rcor1 knock-down cells exhibited delayed migration, remained in the ventricular zone and expressed Sox2 and Tbr2, suggesting the cells had not differentiated from precursor lineages

Rcor2 conditional knock-out (Rcor2 $\left.{ }^{\mathrm{cko}}\right)$ mice had significantly reduced brain sizes, cortical thickness, and structural abnormalities of the brain layers; $R$ cor $2^{\text {cko }}$ mice had reduced numbers of neuronal progenitors and neurons, and increased cell death; the gene knock-out (KO) mice showed significant upregulation of ventral markers and decrease in cortical markers, suggesting CoREST2 regulates the sonic hedgehog signaling pathway

The individual gene knock-out (KO) mice were indistinguishable to the control cohort, combined deletion resulted in severe brain phenotypes and death; Rcor $1 / 2 \mathrm{KO}$ mice had an increased population of proliferating cells, suggesting these mice lacked the mechanism to differentiate precursors into postmitotic neurons and mature oligodendrocytes; CoREST1 and CoREST2 are hypothesized to elicit this function through the formation of a complex with insulinoma-associated 1

CoREST1 interacts with the promoter of $h s p 70$, a gene that encodes heat shock protein 70 (Hsp70); through this interaction, CoREST1 represses both HSF1-dependent and heat-shock-dependent transcriptional activation of hsp70; RCOR1 knock-down resulted in loss of Hsp70 repression, inducing the heat shock response

Rcor2 expression was shown to decrease in an aging mouse model, accompanied by an increase in proinflammatory markers; Rcor2 knock-down further increased inflammatory marker expression

$\begin{array}{cc}\text { Species } & \text { References } \\ \begin{array}{c}\text { Mouse and } \\ \text { human }\end{array} & \begin{array}{c}\text { Yang et al. } \\ \text { (2011) }\end{array}\end{array}$

Chicken Jung et al. (2018)

Mouse

Fuentes et al. (2012)

Mouse

Wang et al. (2016)

Mouse Monaghan et
al. (2017)

Human Gómez et al. (2008)

Mouse

$$
\begin{aligned}
& \text { Alvarez- } \\
& \text { López et } \\
& \text { al. (2014) }
\end{aligned}
$$

CoREST3 to have 3744 peaks and 885 overlap with REST (Yu et al., 2011). Further work is required to identify whether the CoREST proteins binding directly to DNA, the cofactors recruited to the genomic binding site and whether the genes are functionally repressed by the activity of the complex. Through ChIP-on-chip analysis in mouse NSCs, CoREST1 was shown to bind to a broader range of genes (1820 genes) compared with REST (322 genes; Abrajano et al., 2010). Of these genes only 126 were targets of both REST and CoREST1. CoREST1 was identified to target a significantly greater percentage of genes involved in pluripotency such as NANOG/OCT4/ SOX2 network compared with REST (79 compared with eight genes, respectively; Abrajano et al., 2010). Suggesting
CoREST1 has a widespread role in regulating NSC gene networks that is unique to REST. Taken together, both REST and CoREST1 play a role in regulating the switch between NSC self-renewal and neural lineage specification, differentiation, and maturation. Among the genes targeted by REST, $72 \%$ contain known RE1 sites, whereas only $41 \%$ genes targeted by CoREST1 contain known RE1 sites, indicating CoREST1 may repress transcription at additional sites of DNA (Abrajano et al., 2010). In cholinergic, GABAergic, glutamatergic and medium spiny neurons, REST bound to $622,587,481$, and 477 distinct genes, and CoREST1 bound 600, 814, 266, and 967 unique target genes, respectively (Abrajano et al., 2009a). Additionally, 3178 REST and 4060 CoREST1 target genes were 
observed in the two glial cell types, astrocytes and oligodendrocytes (Abrajano et al., 2009b). REST bound to 287 genes specific to astrocytes and 1365 genes specific to oligodendrocytes. CoREST1 was identified to interact with 40 unique targets in astrocytes and 963 genes in oligodendrocytes (Abrajano et al., 2009b). These studies have shown that REST and CoREST1 have the potential to regulate neuronal and glial differentiation, specification, and maintenance via the genes they target. Overall, these findings are suggestive that CoREST1 has a broad, cell type-specific role in neurodevelopment that is distinct and complementary to REST. The DNA sequence each CoREST paralogue targets, the complex formed at these sites and the gene networks regulated are yet to be defined. Further ChIP-on-chip studies with high resolution whole-genome approaches in human tissue will provide insight into the unique and interrelated regulatory networks of REST and CoREST paralogues.

\section{Functional roles of the CoREST family in neurodevelopment}

The molecular mechanisms governing normal development of the brain are yet to be fully elucidated. The regulation of gene expression by transcription factors plays a significant role in the specification and maturation of neurons. Of the three paralogues, CoREST1 has been the most widely studied and is best understood for its role in REST-mediated gene repression. However, recent evidence has shown the CoREST paralogues interact with LSD1 and HDAC1/2 independently of REST and contribute to gene repression (Barrios et al., 2014; Pilotto et al., 2015; Wu et al., 2018). These studies have shifted our understanding that CoREST family act solely as REST corepressors but also have distinct and essential roles in regulating neurodevelopment. The known functions of the CoREST protein family in neurodevelopment is summarized in Table 1. Current knowledge regarding the role of the CoREST proteins has largely been based off animal studies. Rockowitz and Zheng (2015) showed that REST target sites do not completely overlap between human and mouse genomes, with human ESCs having twice as many REST sites as mouse ESCs via ChIP-seq analysis ( $n=8199$ vs $n=4107$ ). From these findings it can be hypothesized that there may also be differences in the genes targeted by each CoREST protein between species. Continued research focusing on the action of the CoREST family will provide insight into the regulatory networks orchestrating neurodevelopment.

It is becoming evident that the CoREST family have unique functions independent to REST, in addition to having distinct roles for each paralogue. Monaghan et al. (2017) showed that Rcor1/Rcor2 knock-out mice had severe deficits in neuronal and glial cell differentiation and a concomitant increase in Rest mRNA levels. Normalization of Rest levels fully restored one of the seven targets that was down regulated (Ce/sr3), the other transcripts were only partially restored (Chrnb2, Trim67, and Unc13a) whereas the remaining three were not rescued (Fam65b, Gad2, and Scrt1). These results indicate that Rcor1 and Rcor2 regulate the switch between proliferation and differentiation in the developing mouse brain in a predominantly Rest-independent manner. In addition, Fuentes et al. (2012) showed Rcor1 knock-down resulted in impaired radial migration of cortical pyramidal neurons in the developing mouse cortex. To confirm the phenotype was mediated by CoREST1, the authors showed overexpression of CoREST1 with a mutated N terminus, to hinder association with Rest, could rescue the migration of neurons in the cerebral cortex. In addition, shRNA knock-down of Rest via electroporation at embryonic day 14 showed no differences in migration when compared with control. These results suggest that CoREST1 regulates pyramidal neuron development independent to Rest in the developing mouse brain. The CoREST paralogues have been shown to have distinct roles in the regulation of pluripotency independent to each other. Overexpression of $R C O R 2$, but not $R C O R 1$, was successful in the reprogramming of induced pluripotent stem cells (Yang et al., 2011). In chicken primordial germ cells, the knock-down of $R C O R 3$ resulted in the upregulation of the pluripotency regulator NANOG, whereas siRNA knock-down of RCOR1 and other chromatin modifying enzymes known to form a complex with REST showed no significant changes in NANOG expression (Jung et al., 2018). Collectively, these studies show that the CoREST family have critical roles during neurodevelopment, that are independent to REST and may have compensating or distinct functions to each paralogue. Further ChIP-on-chip studies with high-resolution whole genome approaches are required to identify the binding sites of each CoREST protein in conjunction with knock-down and overexpression studies to identify the specific pathways and networks regulated, and thus deepen our understanding of the epigenetic mechanisms that govern neurodevelopment.

Our knowledge of the functional roles of the CoREST protein family stems from a heavy reliance on animal models, with the exemption of the study completed by Yang et al. (2011) who was successful in overexpressing RCOR2 to reprogram human stem cells and Gómez et al. (2008) that investigated CoREST1-mediated regulation of the heat shock response in the human embryonic kidney cell line, HEK293. As previously stated, it has been shown that the targets of REST do not overlap between species (Rockowitz and Zheng, 2015), with the same hypothesized for the CoREST paralogues. Thus, human models of neurogenesis, such as human pluripotent stem cells, should be employed to further interrogate the molecular mechanisms regulated by the CoREST family.

\section{The CoREST complex as a potential target for therapeutics}

Aberrant expression or subcellular localization of REST contributes to the disruption of gene regulatory networks and is associated with the pathophysiology of neurodegenerative conditions. It has been hypothesized that targeting REST may help protect from the progression of these conditions. For example, in Huntington's disease (HD), the accumulation of nuclear REST in medium spiny neurons of the striatum results in enhanced repression of $B D N F$ transcription contributing to an increased 
susceptibility to neuronal loss (Zuccato et al., 2003). A potential therapeutic for HD pathogenesis is targeting splicing of REST using antisense oligonucleotides in vitro to reduce the accumulation of nuclear REST (Chen et al., 2017). However, as REST targets the RE1 site in $\sim 2000$ genes in the human genome, the modulation of REST remains a difficult target as it has the potential to have multiple downstream effects. An alternative is to target the LCH complex, for example, using corin, a derivative of a synthesized compound derived from the HDAC1 inhibitor (entinostat) and the LSD1 inhibitor (tranylcypromine analogue) to simultaneously target both components of the LCH complex (Kalin et al., 2018). The dual-hybrid agent has been successfully used as a potential therapeutic in cancer studies by reducing the proliferation of mouse melanoma cells through blocking the active sites of HDAC1 and LSD1 of the LCH complex (Kalin et al., 2018). Synaptic dysfunction is a common in many neurodegenerative diseases including $A D$, with HDACs involved in regulating synaptogenesis and synaptic plasticity (Fuller et al., 2019). HDAC inhibitors have been trialed to improve synaptic growth and function, but have been limited due to their off-target effects and dose-limiting hematologic toxicities. Fuller et al. (2019) used the CoREST-selective HDAC inhibitor Rodin-A in a mouse model and were successful in increasing spine density, expression of synaptic proteins and long-term potentiation at suitable doses to allow for chronic treatment. This study has shown that selectively targeting the CoREST complex, and not the Sin3, NCoR, and NuRD complexes, offers a promising therapeutic for synaptopathies and that the CoREST complex is a key regulator of synaptic growth and function (Fuller et al., 2019).

The regulatory mechanisms governing CoREST protein expression and function remain largely unknown. Sáez et al. (2018) have described a possible mode of regulation of the CoREST proteins via the interaction with protein inhibitor of activated STAT (PIASy) and SUMOylation which may control cell fate determination during development. Abrajano et al. (2010) proposed a mechanism in which CoREST1 regulates its own expression by binding to the gene Senp1, which encodes for a small ubiquitin-like modifier (SUMO)-specific protease and has been previously shown to inhibit CoREST1 activity (Muraoka et al., 2008). Future investigation into the regulatory networks of each CoREST paralogue will be crucial in understanding the processes of neurodevelopment and may provide potential therapeutic targets for neurodegenerative conditions.

\section{Summary}

In summary, REST has been well documented for its function in neuronal differentiation and maturation, with new evidence emerging of a potential neuroprotective role in neurodegeneration. More focus needs to be dedicated to the CoREST family, as the importance of CoREST-mediated gene repression during neurodevelopment is continuing to grow in the field. It is clear that there are differences in the target genes between CoREST1 and REST in different neuronal and glial cell types, suggesting that each paralogue may play distinct and important roles in neurodevelopment. Future studies focused on the target genes, extensive expression profile and regulatory networks of the CoREST paralogues in different human neural and glial cell types will deepen our understanding of the tightly regulated molecular mechanisms of neurogenesis and normal brain development. It is clear that CoREST proteins are essential for proper neurodevelopment and dysfunction of these regulatory mechanisms are potentially linked to the progression of neurodegenerative conditions. Therefore, the CoREST family have a broader function outside of acting solely as a REST corepressor but are also independent and critical regulators of neurodevelopment.

\section{References}

Abrajano JJ, Qureshi IA, Gokhan S, Zheng D, Bergman A, Mehler MF (2009a) REST and CoREST modulate neuronal subtype specification, maturation and maintenance. PLoS One 4:e7936.

Abrajano JJ, Qureshi IA, Gokhan S, Zheng D, Bergman A, Mehler MF (2009b) Differential deployment of REST and CoREST promotes glial subtype specification and oligodendrocyte lineage maturation. PLoS One 4:e7665.

Abrajano JJ, Qureshi IA, Gokhan S, Molero AE, Zheng D, Bergman A, Mehler MF (2010) Corepressor for element-1-silencing transcription factor preferentially mediates gene networks underlying neural stem cell fate decisions. Proc Natl Acad Sci USA 107:16685-16690.

Allfrey V, Faulkner R, Mirsky A (1964) Acetylation and methylation of histones and their possible role in the regulation of RNA synthesis. Proc Natl Acad Sci USA 51:786-794.

Alvarez-López MJ, Molina-Martínez $P$, Castro-Freire M, CosínTomás $M$, Cristòfol $R$, Párrizas $M$, Escorihuela RM, Pallàs $M$, Sanfeliu C, Kaliman P (2014) Rcor2 underexpression in senescent mice: A target for inflammaging? J Neuroinflammation 11:126.

Andrés ME, Burger C, Peral-Rubio MJ, Battaglioli E, Anderson ME, Grimes J, Dallman J, Ballas N, Mandel G (1999) CoREST: A functional corepressor required for regulation of neural-specific gene expression. Proc Natl Acad Sci USA 96:9873-9878.

Armisén R, Fuentes R, Olguín P, Cabrejos ME, Kukuljan M (2002) Repressor element-1 silencing transcription/neuron-restrictive silencer factor is required for neural sodium channel expression during development of Xenopus. J Neurosci 22:8347-8351.

Ashton NJ, Hye A, Leckey CA, Jones AR, Gardner A, Elliott C, Wetherell JL, Lenze EJ, Killick R, Marchant NL (2017) Plasma REST: A novel candidate biomarker of Alzheimer's disease is modified by psychological intervention in an at-risk population. Transl Psychiatry 7:e1148.

Ballas N, Grunseich C, Lu DD, Speh JC, Mandel G (2005) REST and its corepressors mediate plasticity of neuronal gene chromatin throughout neurogenesis. Cell 121:645-657.

Barrios ÁP, Gómez AV, Sáez JE, Ciossani G, Toffolo E, Battaglioli E, Mattevi A, Andrés ME (2014) Differential properties of transcriptional complexes formed by the CoREST family. Mol Cell Biol 34:2760-2770.

Battaglioli E, Andrés ME, Rose DW, Chenoweth JG, Rosenfeld MG, Anderson ME, Mandel G (2002) REST repression of neuronal genes requires components of the hSWI. SNF complex. J Biol Chem 277:41038-41045.

Boyer LA, Langer MR, Crowley KA, Tan S, Denu JM, Peterson CL (2002) Essential role for the SANT domain in the functioning of multiple chromatin remodeling enzymes. Mol Cell 10:935-942.

Boyer LA, Latek RR, Peterson CL (2004) The SANT domain: A unique histone-tail-binding module? Nat Rev Mol Cell Biol 5:158-163.

Boyer LA, Lee TI, Cole MF, Johnstone SE, Levine SS, Zucker JP, Guenther MG, Kumar RM, Murray HL, Jenner RG, Gifford DK, 
Melton DA, Jaenisch R, Young RA (2005) Core transcriptional regulatory circuitry in human embryonic stem cells. Cell 122:947-956.

Bruce AW, Donaldson IJ, Wood IC, Yerbury SA, Sadowski MI, Chapman M, Göttgens B, Buckley NJ (2004) Genome-wide analysis of repressor element 1 silencing transcription factor/neuron-restrictive silencing factor (REST/NRSF) target genes. Proc Natl Acad Sci USA 101:10458-10463.

Calderone A, Jover T, Noh KM, Tanaka H, Yokota H, Lin Y, Grooms SY, Regis R, Bennett MVL, Zukin RS (2003) Ischemic insults derepress the gene silencer REST in neurons destined to die. $J$ Neurosci 23:2112-2121.

Chen GL, Ma Q, Goswami D, Shang J, Miller GM (2017) Modulation of nuclear REST by alternative splicing: A potential therapeutic target for Huntington's disease. J Cell Mol Med 21:2974-2984.

Chong JA, Tapia-Ramírez J, Kim S, Toledo-Aral JJ, Zheng Y, Boutros MC, Altshuller YM, Frohman MA, Kraner SD, Mandel G (1995) REST: A mammalian silencer protein that restricts sodium channel gene expression to neurons. Cell 80:949-957.

Conforti P, Zuccato C, Gaudenzi G, leraci A, Camnasio S, Buckley NJ, Mutti C, Cotelli F, Contini A, Cattaneo E (2013) Binding of the repressor complex REST-mSIN3b by small molecules restores neuronal gene transcription in Huntington's disease models. J Neurochem 127:22-35.

Ding Z, Gillespie LL, Paterno GD (2003) Human MI-ER1 alpha and beta function as transcriptional repressors by recruitment of histone deacetylase 1 to their conserved ELM2 domain. Mol Cell Biol 23:250-258.

Forneris F, Binda C, Adamo A, Battaglioli E, Mattevi A (2007) Structural basis of LSD1-CoREST selectivity in histone $\mathrm{H} 3$ recognition. J Biol Chem 282:20070-20074.

Fuentes P, Cánovas J, Berndt FA, Noctor SC, Kukuljan M (2012) CoREST/LSD1 control the development of pyramidal cortical neurons. Cereb Cortex 22:1431-1441.

Fuks F, Hurd PJ, Wolf D, Nan X, Bird AP, Kouzarides T (2003) The methyl-CpG-binding protein MeCP2 links DNA methylation to histone methylation. J Biol Chem 278:4035-4040.

Fuller NO, Pirone A, Lynch BA, Hewitt MC, Quinton MS, McKee TD, Ivarsson M (2019) CoREST complex-selective histone deacetylase inhibitors show prosynaptic effects and an improved safety profile to enable treatment of synaptopathies. ACS Chem Neurosci 10:1729-1743.

Gao Z, Ure K, Ding P, Nashaat M, Yuan L, Ma J, Hammer RE, Hsieh $J$ (2011) The master negative regulator REST/NRSF controls adult neurogenesis by restraining the neurogenic program in quiescent stem cells. J Neurosci 31:9772-9786.

Gómez AV, Galleguillos D, Maass JC, Battaglioli E, Kukuljan M, Andrés ME (2008) CoREST represses the heat shock response mediated by HSF1. Mol Cell 31:222-231.

Grimes JA, Nielsen SJ, Battaglioli E, Miska EA, Speh JC, Berry DL, Atouf F, Holdener BC, Mandel G, Kouzarides T (2000) The co-repressor mSin3A is a functional component of the REST-CoREST repressor complex. J Biol Chem 275:9461-9467.

Gupta SK, Gressens P, Mani S (2009) NRSF downregulation induces neuronal differentiation in mouse embryonic stem cells. Differentiation 77:19-28.

Huang D, Li Q, Wang Y, Liu Z, Wang Z, Li H, Wang J, Su J, Ma Y, Yu $M$, Fei J, Huang $F$ (2019) Brain-specific NRSF deficiency aggravates dopaminergic neurodegeneration and impairs neurogenesis in the MPTP mouse model of Parkinson's disease. Aging (Albany NY) 11:3280-3297.

Huang Y, Myers SJ, Dingledine R (1999) Transcriptional repression by REST: Recruitment of Sin3A and histone deacetylase to neuronal genes. Nat Neurosci 2:867-872.

Inui K, Zhao Z, Yuan J, Jayaprakash S, Le LTM, Drakulic S, Sander B, Golas MM (2017) Stepwise assembly of functional C-terminal REST/NRSF transcriptional repressor complexes as a drug target. Protein Sci 26:997-1011.

Johnson R, Gamblin RJ, Ooi L, Bruce AW, Donaldson IJ, Westhead DR, Wood IC, Jackson RM, Buckley NJ (2006) Identification of the
REST regulon reveals extensive transposable element-mediated binding site duplication. Nucleic Acids Res 34:3862-3877.

Jung HG, Hwang YS, Park YH, Cho HY, Rengaraj D, Han JY (2018) Role of epigenetic regulation by the REST/CoREST/HDAC corepressor complex of moderate NANOG expression in chicken primordial germ cells. Stem Cells Dev 27:1215-1225.

Kalin JH, Wu M, Gomez AV, Song Y, Das J, Hayward D, Adejola N, Wu M, Panova I, Chung HJ, Kim E, Roberts HJ, Roberts JM, Prusevich $P$, Jeliazkov JR, Roy Burman SS, Fairall L, Milano C, Eroglu A, Proby $\mathrm{CM}$, et al. (2018) Targeting the CoREST complex with dual histone deacetylase and demethylase inhibitors. Nat Commun 9:53.

Kawamura M, Sato S, Matsumoto G, Fukuda T, Shiba-Fukushima K, Noda S, Takanashi M, Mori N, Hattori N (2019) Loss of nuclear REST/NRSF in aged-dopaminergic neurons in Parkinson's disease patients. Neurosci Lett 699:59-63.

Kim HJ, Denli AM, Wright R, Baul TD, Clemenson GD, Morcos AS, Zhao C, Schafer ST, Gage FH, Kagalwala MN (2015) REST regulates non-cell-autonomous neuronal differentiation and maturation of neural progenitor cells via secretogranin II. J Neurosci 35:14872-14884.

Kuwabara T, Hsieh J, Nakashima K, Taira K, Gage FH (2004) A small modulatory dsRNA specifies the fate of adult neural stem cells. Cell 116:779-793.

Lee MG, Wynder C, Cooch N, Shiekhattar R (2005) An essential role for CoREST in nucleosomal histone 3 lysine 4 demethylation. Nature 437:432-435.

Lu T, Aron L, Zullo J, Pan Y, Kim H, Chen Y, Yang T-H, Kim H-M, Drake D, Liu XS, Bennett DA, Colaiácovo MP, Yankner BA (2014) REST and stress resistance in ageing and Alzheimer's disease. Nature 507:448-454.

Lunyak VV, Burgess R, Prefontaine GG, Nelson C, Sze S-H, Chenoweth J, Schwartz P, Pevzner PA, Glass C, Mandel G, Rosenfeld MG (2002) Corepressor-dependent silencing of chromosomal regions encoding neuronal genes. Science 298:1747-1752.

Mandel G, Fiondella CG, Covey MV, Lu DD, LoTurco JJ, Ballas N (2011) Repressor element 1 silencing transcription factor (REST) controls radial migration and temporal neuronal specification during neocortical development. Proc Natl Acad Sci USA 108:16789-16794.

Marchler-Bauer A, Bo Y, Han L, He J, Lanczycki CJ, Lu S, Chitsaz F, Derbyshire MK, Geer RC, Gonzales NR, Gwadz M, Hurwitz DI, Lu F, Marchler GH, Song JS, Thanki N, Wang Z, Yamashita RA, Zhang D, Zheng C, et al. (2017) CDD/SPARCLE: Functional classification of proteins via subfamily domain architectures. Nucleic Acids Res 45:D200-D203.

McGann JC, Oyer JA, Garg S, Yao H, Liu J, Feng X, Liao L, Yates JR 3rd, Mandel G (2014) Polycomb- and REST-associated histone deacetylases are independent pathways toward a mature neuronal phenotype. Elife 3:e04235.

Meyer K, Feldman HM, Lu T, Drake D, Lim ET, Ling K-H, Bishop NA, Pan Y, Seo J, Lin Y-T, Su SC, Church GM, Tsai L-H, Yankner BA (2019) REST and neural gene network dysregulation in iPSC models of Alzheimer's disease. Cell Rep 26:1112-1127.e9.

Monaghan CE, Nechiporuk T, Jeng S, McWeeney SK, Wang J, Rosenfeld MG, Mandel G (2017) REST corepressors RCOR1 and RCOR2 and the repressor INSM1 regulate the proliferation-differentiation balance in the developing brain. Proc Natl Acad Sci USA 114:E406-E415.

Muraoka A, Maeda A, Nakahara N, Yokota M, Nishida T, Maruyama T, Ohshima T (2008) Sumoylation of CoREST modulates its function as a transcriptional repressor. Biochem Biophys Res Commun 377:1031-1035.

Nair VD, Ge Y, Balasubramaniyan N, Kim J, Okawa Y, Chikina M, Troyanskaya O, Sealfon SC (2012) Involvement of histone demethylase LSD1 in short-time-scale gene expression changes during cell cycle progression in embryonic stem cells. Mol Cell Biol 32:4861-4876

Naruse Y, Aoki T, Kojima T, Mori N (1999) Neural restrictive silencer factor recruits $\mathrm{mSin} 3$ and histone deacetylase complex to repress neuron-specific target genes. Proc Natl Acad Sci USA 96:13691-13696. 
Nechiporuk T, McGann J, Mullendorff K, Hsieh J, Wurst W, Floss T, Mandel G (2016) The REST remodeling complex protects genomic integrity during embryonic neurogenesis. Elife 5:e09584.

Ooi L, Belyaev ND, Miyake K, Wood IC, Buckley NJ (2006) BRG1 chromatin remodeling activity is required for efficient chromatin binding by repressor element 1-silencing transcription factor (REST) and facilitates REST-mediated repression. J Biol Chem 281:38974-38980.

Palm K, Metsis M, Timmusk T (1999) Neuron-specific splicing of zinc finger transcription factor REST/NRSF/XBR is frequent in neuroblastomas and conserved in human, mouse and rat. Mol Brain Res 72:30-39

Paquette AJ, Perez SE, Anderson DJ (2000) Constitutive expression of the neuron-restrictive silencer factor (NRSF)/REST in differentiating neurons disrupts neuronal gene expression and causes axon pathfinding errors in vivo. Proc Natl Acad Sci USA 97:12318-12323.

Pilotto S, Speranzini V, Tortorici M, Durand D, Fish A, Valente S, Forneris F, Mai A, Sixma TK, Vachette P, Mattevi A (2015) Interplay among nucleosomal DNA, histone tails, and corepressor CoREST underlies LSD1-mediated H3 demethylation. Proc Natl Acad Sci USA 112:2752-2757.

Rockowitz S, Zheng D (2015) Significant expansion of the REST/ NRSF cistrome in human versus mouse embryonic stem cells: Potential implications for neural development. Nucleic Acids Res 43:5730-5743.

Rodenas-Ruano A, Chávez AE, Cossio MJ, Castillo PE, Zukin RS (2012) REST-dependent epigenetic remodeling promotes the developmental switch in synaptic NMDA receptors. Nat Neurosci 15:1382-1390.

Roopra A, Qazi R, Schoenike B, Daley TJ, Morrison JF (2004) Localized domains of G9a-mediated histone methylation are required for silencing of neuronal genes. Mol Cell 14:727-738.

Sáez JE, Gómez AV, Barrios ÁP, Parada GE, Galdames L, González M, Andrés ME (2015) Decreased expression of CoREST1 and CoREST2 together with LSD1 and HDAC1/2 during neuronal differentiation. PLoS One 10:e0131760.

Sáez JE, Arredondo C, Rivera C, Andrés ME (2018) PIAS $\gamma$ controls stability and facilitates SUMO-2 conjugation to CoREST family of transcriptional co-repressors. Biochem J 475:1441-1454.

Satoh JI, Kawana N, Yamamoto Y (2013) ChIP-Seq data mining: Remarkable differences in NRSF/REST target genes between human ESC and ESC-derived neurons. Bioinform Biol Insights 7:357-368.

Schoenherr C, Anderson D (1995) The neuron-restrictive silencer factor (NRSF): A coordinate repressor of multiple neuron-specific genes. Science 267:1360-1363.

Shi YJ, Matson C, Lan F, Iwase S, Baba T, Shi Y (2005) Regulation of LSD1 histone demethylase activity by its associated factors. Mol Cell 19:857-864.

Shiio Y, Eisenman RN (2003) Histone sumoylation is associated with transcriptional repression. Proc Natl Acad Sci USA 100:13225-13230.

Singh Y, Leinonen H, Fazaludeen F, Jaronen M, Guest D, Buckley N, Byts N, Oksa P, Jalkanen K, Iqbal I, Huuskonen M, Savchenko E, Keksa-Goldsteine V, Chew S, Myllyharju J, Tanila H, Ooi L, Koistinaho J, Kanninen KM, Malm T (2019) Loss of Cln5 leads to altered Gad1 expression and deficits in interneuron development in mice. Hum Mol Genet 28:3309-3322.

Solari F, Bateman A, Ahringer J (1999) The Caenorhabditis elegans genes egl-27 and egr-1 are similar to MTA1, a member of a chromatin regulatory complex, and are redundantly required for embryonic patterning. Development 126:2483-2494.

Song Z, Zhu T, Zhou X, Barrow P, Yang W, Cui Y, Yang L, Zhao D (2016) REST alleviates neurotoxic prion peptide-induced synaptic abnormalities, neurofibrillary degeneration and neuronal death partially via LRP6-mediated Wnt- $\beta$-catenin signaling. Oncotarget 7:12035-12052.

Song Z, Yang W, Zhou X, Yang L, Zhao D (2017a) Lithium alleviates neurotoxic prion peptide-induced synaptic damage and neuronal death partially by the upregulation of nuclear target REST and the restoration of Wnt signaling. Neuropharmacology 123:332-348.

Song Z, Shah SZA, Yang W, Dong H, Yang L, Zhou X, Zhao D (2017b) Downregulation of the repressor element 1-silencing transcription factor (REST) is associated with Akt-mTOR and Wnt- $\beta$-catenin signaling in prion diseases models. Front Mol Neurosci 10:128.

Su X, Kameoka S, Lentz S, Majumder S (2004) Activation of REST/ NRSF target genes in neural stem cells is sufficient to cause neuronal differentiation. Mol Cell Biol 24:8018-8025.

Sun ZW, Allis CD (2002) Ubiquitination of histone H2B regulates $\mathrm{H} 3$ methylation and gene silencing in yeast. Nature 418:104-108.

Suo H, Wang P, Tong J, Cai L, Liu J, Huang D, Huang L, Wang Z, Huang Y, Xu J, Ma Y, Yu M, Fei J, Huang F (2015) NRSF is an essential mediator for the neuroprotection of trichostatin A in the MPTP mouse model of Parkinson's disease. Neuropharmacology 99:67-78.

Tabuchi A, Yamada T, Sasagawa S, Naruse Y, Mori N, Tsuda M (2002) REST4-mediated modulation of REST/NRSF-silencing function during BDNF gene promoter activation. Biochem Biophys Res Commun 290:415-420.

Tachibana M, Sugimoto K, Fukushima T, Shinkai Y (2001) SET-domain containing protein, G9a, is a novel lysine-preferring mammalian histone methyltransferase with hyperactivity and specific selectivity to lysines 9 and 27 of histone H3. J Biol Chem 276:25309-25317.

UniProt Consortium (2018) UniProt: The universal protein knowledgebase. Nucleic Acids Res 46:2699-2699.

Upadhyay G, Chowdhury AH, Vaidyanathan B, Kim D, Saleque S (2014) Antagonistic actions of Rcor proteins regulate LSD1 activity and cellular differentiation. Proc Natl Acad Sci USA 111:8071-8076.

Wang Y, Wu Q, Yang P, Wang C, Liu J, Ding W, Liu W, Bai Y, Yang Y, Wang H, Gao S, Wang X (2016) LSD1 co-repressor Rcor2 orchestrates neurogenesis in the developing mouse brain. Nat Commun 7:10481

Wei Y, Yu L, Bowen J, Gorovsky MA, Allis CD (1999) Phosphorylation of histone $\mathrm{H} 3$ is required for proper chromosome condensation and segregation. Cell 97:99-109.

Wu M, Hayward D, Kalin JH, Song Y, Schwabe JW, Cole PA (2018) Lysine-14 acetylation of histone $\mathrm{H} 3$ in chromatin confers resistance to the deacetylase and demethylase activities of an epigenetic silencing complex. Elife 7:e37231.

Yang M, Gocke CB, Luo X, Borek D, Tomchick DR, Machius M, Otwinowski Z, Yu H (2006) Structural basis for CoREST-dependent demethylation of nucleosomes by the human LSD1 histone demethylase. Mol Cell 23:377-387.

Yang P, Wang Y, Chen J, Li H, Kang L, Zhang Y, Chen S, Zhu B, Gao $S$ (2011) RCOR2 is a subunit of the LSD1 complex that regulates ESC property and substitutes for SOX2 in reprogramming somatic cells to pluripotency. Stem Cells 29:791-801.

You A, Tong JK, Grozinger CM, Schreiber SL (2001) CoREST is an integral component of the CoREST- human histone deacetylase complex. Proc Natl Acad Sci USA 98:1454-1458.

Yu HB, Johnson R, Kunarso G, Stanton LW (2011) Coassembly of REST and its cofactors at sites of gene repression in embryonic stem cells. Genome Res 21:1284-1293.

Zhang F, Gigout S, Liu Y, Wang Y, Hao H, Buckley NJ, Zhang H, Wood IC, Gamper N (2019) Repressor element 1-silencing transcription factor drives the development of chronic pain states. Pain 160:2398-2408.

Zuccato C, Tartari M, Crotti A, Goffredo D, Valenza M, Conti L, Cataudella T, Leavitt BR, Hayden MR, Timmusk T, Rigamonti D, Cattaneo E (2003) Huntingtin interacts with REST/NRSF to modulate the transcription of NRSE-controlled neuronal genes. Nat Genet 35:76-83.

Zuccato C, Belyaev N, Conforti P, Ooi L, Tartari M, Papadimou E, MacDonald M, Fossale E, Zeitlin S, Buckley N, Cattaneo E (2007) Widespread disruption of repressor element-1 silencing transcription factor/neuron-restrictive silencer factor occupancy at its target genes in Huntington's disease. J Neurosci 27:6972-6983. 